can entertain a doubt as to its being the most absurd and dis. honest form of quackery and imposture ever foisted upon the public. But the fact, to which we would direct attention, is, the assumption of the Royal Court of Guernsey to grant licences to practise medicine and surgery. Were such licences limited to duly qualified practitioners, no blame might attach to this stretch of power, as the public would thereby be protected from deception and fraud; but the incompetency of the Court to judge of the authenticity and validity of assumed diplomas is proved by the fact, that unqualified individuals, and uneducated ai.venturers, on presentation of alleged diplomas from the "British College of Heal:h," \&c., or certificates, as "Officicrs de Santé," have obtained leave to practise. And Mr. OzıNve, in 18t0, on an alleged diploma, as "docteur en médecine" of the faculty of Paris, only, procured from the said court a licence to practise surgery. Nevertheless, with additional inconsistency, a licence to practise in Guernsey was refused a few months since to a graduate of Cambridge.

It is high time that the Apothecaries' Act should be registered in Guerrsey, as a protection to those who have voluntarily pursued the course of study prescribed-to those who have undergone the trouble and expense of examinations before the Medical and Surgical Colleges, and Society of Apothecaries, and who, from their education and respect. ability, are entitled to be protected from imposture and quackery.

\section{A Anirror}

OF THE PRACTICE OF

\section{MEDICINE AND SURGERY IN THE \\ HOSPITALS OF LONDON.}

\section{GuY's Hospital. \\ Amputation at the Shoulder-joint.}

(Under the care of Mr. BransBY Cooper.)

WE recently witnessed this important operation performed, by Mr. Bransby Cooper, on a woman, twenty-five years of age, who had been suffering for years from necrosis of the humerus. The history of the case, as noted down by Mr. Stocker, the dresser of the patient, is as follows:-Harriet $\mathbf{S}-$, a servant, of dark complexion, was admitted into Dorcas ward, in May, 1848, under the care of Mr. Aston Key. She stated, that for no less than twelve years she had been suffering from necrosis of the humerus, and that the disease had originally been caused by a fracture of that bone. She had been a patient in Guy's Hospital eight years previous to her admission in 1848, when Mr. Key had operated upon her, and had removed two pieces of dead bone. During this interval she had otherwise enjoyed good health, and the arm being now carefully examined, Mr. Key determined to remove the necrosed portions of bone. The patient, having been placed under the influence of chloroform, a crucial incision was made over the upper part of the external condyloid ridge of the humerus, and the integuments dissected back. The bone being thus laid quite bare, several sequestra were removed with the chisel and hammer.

One month after this operation, the wound was quite healed, som : more pieces of bone having come away during the suppurative stage. She remained in the hospital for two months after cicatrization, and $\mathbf{M r}$. Key, then finding that the upper part of the humerus was also diseased, operated in that region likewise, but removed only a very small portion of necrosed bone. She soon recovered from this second operation, and went out nearly well.

The patient had, however, as is but too often the case, ex. perienced only temporary relief, and she applied again for admission in December, 1849 , when she was placed under the care of Mr. Bransby Cooper. She now complained of great pain in the arm, increasing so much at night as to destroy her rest completely. The limb presented a number of apertures of simuses, with the usual inverted edges and a light-yellow discharge, and the probe introduced into one of them in the upper part of the arm readily detocted a moveable piece of necrosed bone. One month after this last admission, Mr. Cooper removed a sequestrum, about three inches in length, from the superior portion of the humerns, and the patient expressed herself considerably relieved by this measure; the pain diminished notably, and she remained in a tolerable condition for about three weeks, when the arm and shoulder became again excessively painful, so much so as to entirely prevent her from sleeping. $\mathrm{Mr}$. Cooper perceiving that the girl's health was being undermined by this protracted affection, and fearful that the disease might extend to the joint, proposed amputation at the scapulo-humeral articulation, and the patient very readily submitted to the necessity of the case.

She was brought into the theatre on the 12 th of March, and refused to inhale the chloroform, stating that she knew she must undergo the operation, and would have the fortitude to go through it. Though such courage calls for great admiration, it is alnost a pity that pain should be endured when we possess such a ready means of avoiding it. The ratient being seated, Mr. Cooper made his first incision, with a large-sized knife, in a longitudinal direction, commencing a little externally to the acromion, and descending in a curved manner to about four inches from that process near the in. sertion of the deltoid. The second incision was made with the left hand, and began a little internally to the acromion, run downwards, and terminated at the extremity of the first cut. The deltoid being thus comprised by these two incisions, was rapidly dissected upwards towards the head of the humerus, and the flap being held by an assistant, the tendon of the biceps, those attached to the head of the bone, and the capsule, were diviled with a similar celerity. The humerus having been disarticulated, Mr. Cooper inquired whether the assistants were ready, and with one sweep of the knife divided the soft parts situated interually. From the beginning of the operation, Mr. Birkett exercised steady compression on the subclavian artery with a padded key, and the flap containing the axillary artery being seized immediately the muscles were divided, the circulation was thus so completely commanded that the hæmorrhage was very trifling. The axillary artery was immediately secured, with a few other vessels of lesser importance, the flap brought into contact with the parts next to the trunk, the wound lightly dressed, and the patient carefully removed to her bed. From the remarkable celerity with which this amputation was performed, we should be inclined constantly to give the preference to this mode of operating. There seems to be more certainty in making a square or circular flap at the cushion of the shoulder than to thrust the double edged knife through the base of the deltoid, and run it downwards to the insertion of the muscle. The nature of the injury for which the operation is performed will very often leave the surgeon no choice, and oblige him to take a flap where he can; but when there is a choice, the advantage seems to lie on the method which Mr. Cooper adopted. The patient has progressed very favourably up to this day; most of the ligatures have come away, the wound has healed by first intention, and the stump is of a very regular form. On examining the limb, it was fonnd, that from the condyles up to the centre of the shaft, the bone was pretty healthy; the rest of the shaft was perforated by three large holes, in one of which lay a loose sequestrum. The head of the humerus was severely attacked, the cartilage partly gone, and the neck almost, destroyed. The two upper perforations communicated with each other, the medullary canal being thereby much encroached upon. The glenoid cavity was, however, free from disease.

\section{Royal Free Hospitat.}

Endocarditis and Cirrhosis of the Liver.

('inder the care of Dr. Hedtr.)

Dr. Heale had lately in his wards a patient whose case afforded various features of interest. The patient was only twenty-three years of age, and presented a series of affections 